Journal of Systems Science and Information

Feb., 2017, Vol. 5, No. 1, pp. 21-33

DOI: $10.21078 /$ JSSI-2017-021-13

\title{
Optimal Consumption and Portfolio Decision with Heston's SV Model Under HARA Utility Criterion
}

\author{
Chunfeng WANG \\ College of Management and Economics, Tianjin University, Tianjin 300072, China \\ E-mail: cfwang@tju.edu.cn \\ Hao CHANG \\ College of Management and Economics, Tianjin University, Tianjin 300072, China; School of \\ Science, Tianjin Polytechnic University, Tianjin 300387, China \\ E-mail: ch8683897@126.com \\ Zhenming FANG \\ College of Management and Economics, Tianjin University, Tianjin 300072, China \\ E-mail: zmfang@tju.edu.cn
}

\begin{abstract}
This paper studies the optimal consumption-investment strategy with Heston's stochastic volatility (SV) model under hyperbolic absolute risk aversion (HARA) utility criterion. The financial market is composed of a risk-less asset and a risky asset, whose price process is supposed to be driven by Heston's SV model. The risky preference of the individual is assumed to satisfy HARA utility, which recovers power utility, exponential utility and logarithm utility as special cases. HARA utility is of general framework in the utility theory and is seldom studied in the existing literatures. Legendre transform-dual technique along with stochastic dynamic programming principle is presented to deal with our problem and the closed-form solution to the optimal consumption-investment strategy is successfully obtained. Finally, some special cases are derived in detail.
\end{abstract}

Keywords investment-consumption problem; Heston model; HARA preference; Legendre transformdual theory; closed-form solution

\section{Introduction}

The investment-consumption problems were originated from the seminal papers of Merton ${ }^{[1,2]}$, who used stochastic optimal control theory to study portfolio selection problems for the first time. Since then, there are thousands of millions of research results on the investment-consumption models, for example, Fleming and Hernandez-hernandez ${ }^{[3]}$ and Chacko and Viceira ${ }^{[4]}$ respectively studied the investment-consumption problems with the Heston model under the different market assumptions. In the decade years, some scholars began to focus on the time-inconsistent

Received April 5, 2016, accepted May 19, 2016

Supported by the National Natural Science Foundation of China (71671122), China Postdoctoral Science Foundation Funded Project (2014M560185, 2016T90203), Humanities and Social Science Research Fund of Ministry of Education of China (11YJC790006, 16YJA790004) and Tianjin Natural Science Foundation of China (15JCQNJC04000) 
investment-consumption problems, the interested readers can refer to the works of Ekeland and Pirvu $^{[5]}$, Pirvu and Zhang ${ }^{[6]}$, Zhao, et al. ${ }^{[7]}$, Zou, et al. ${ }^{[8]}$, and so on. Those papers mentioned above greatly enriched and extended the original investment-consumption models, but these research results were achieved under the assumption of power utility. Compared with power utility, HARA utility is a more general utility function, which recovers power utility, exponential utility and logarithm utility as special cases.

In the last few years, some scholars began to concern the portfolio selection problems with HARA utility. Fortunately, some important results were achieved by using Legendre transformdual theory. For example, Jung and Kim ${ }^{[9]}$ studied the portfolio selection problem under a constant elasticity of variance model $(\mathrm{CEV})$ model. Later, Chang and Rong ${ }^{[10]}$ investigated an investment-consumption problem under HARA utility criterion. Chang et al. ${ }^{[11]}$ focused on an asset-liability management problem with stochastic affine interest rate under HARA utility. From these literatures, we found that Legendre transform-dual theory along with stochastic dynamic programming principle is an effective methodology in dealing with the portfolio selection problems under HARA utility. In addition, there are two advantages in application of Legendre transform-dual theory. One is that Legendre transform-dual theory can transform the nonlinear partial differential equation into a linear partial differential equation, which is very easy to solve. The other is that the boundary condition of dual equation under HARA utility is linear, which make the structure of the solution be easily conjectured. Understanding more information on Legendre transform-dual theory, one can read the papers of Jonsson and Sircar $^{[12]}$ and Gao ${ }^{[13]}$.

In the practical investments, the volatility of stock price is always stochastic. The Heston model ${ }^{[14]}$ is an important and simple one in the models of stochastic volatility. In recent years, the portfolio selection problems with Hestion's SV model have attracted increasing attentions of many scholars. On newly research results under the Heston model, the interested readers may refer to the papers of $\mathrm{Li}$, et al. ${ }^{[15]}$, Yi, et al. ${ }^{[16]}$, Zhao, et al. ${ }^{[17]}$, Li, et al. ${ }^{[18]}$, and $\mathrm{A}$ and $\mathrm{Li}^{[19]}$. As far as we know, the optimal consumption-investment strategy with the Heston model under HARA preference hasn't been reported in the existing literatures.

In this paper, we assume the risk preference of individuals to satisfy HARA utility and study the optimal consumption and portfolio decision with Heston's stochastic volatility model. The financial market is composed of a risk-less asset and a risky asset, whose price dynamics is supposed to be driven by the Heston model. Legendre transform-dual theory is used to change the original HJB equation into its dual equation, whose boundary condition is linear under HARA utility. It displays that it is very easy to conjecture the structure of the solution to the dual equation. Finally, we obtain the explicit solution to the optimal consumption-investment strategy. In addition, some special cases are derived in detail. In conclusion, there are three main contributions: (i) The optimal consumption and portfolio decision with the Heston model is studied; (ii) The explicit solution under HARA utility are successfully obtained; (iii) Legendre transform-dual theory is used to deal with our model.

The remainder of this paper is organized as follows. Section 2 describes the problem framework of the optimal consumption and portfolio decision with Heston's SV model and gives the HARA utility function. Section 3 uses Legendre transform-dual theory along with stochastic 
dynamic programming principle to derive the dual equation to the original HJB equation. The optimal consumption and portfolio decision under HARA utility and some special cases are derived in Section 4. Finally, some conclusions are drawn in Section 5.

\section{Problem Formulation}

Assume that $W_{1}(t)$ is a one-dimensional standard Brownian motion defined on complete probability space $\left(\Omega, \mathscr{F},\left\{\mathscr{F}_{t}\right\}_{t \in[0, T]}, \mathbb{P}\right)$, where $\left\{\mathscr{F}_{t}\right\}_{t \in[0, T]}$ is the information collection generated by random resource $W_{1}(t)$. $[0, T]$ is a fixed time horizon.

Suppose that the financial market consists of two assets traded continuously. One asset is the risk-free asset, whose price is denoted by $S_{0}(t)$ at time $t$. Then $S_{0}(t)$ satisfies

$$
\mathrm{d} S_{0}(t)=r S_{0}(t) \mathrm{d} t, \quad S_{0}(0)=s_{0}>0,
$$

where $r>0$ is constant risk-free interest rate.

The other asset is a stock, whose price process is denoted by $S_{1}(t)$ at time $t$. Then $S_{1}(t)$ is supposed to be driven by Heston's stochastic volatility model ${ }^{[14]}$

$$
\begin{aligned}
& \mathrm{d} S_{1}(t)=S_{1}(t)\left[(r+\lambda L(t)) \mathrm{d} t+\sqrt{L(t)} \mathrm{d} W_{1}(t)\right], \quad S_{1}(0)=s_{1}>0, \\
& \mathrm{~d} L(t)=k(\theta-L(t)) \mathrm{d} t+\sigma \sqrt{L(t)} \mathrm{d} W_{1}(t), \quad L_{1}(0)=l_{0}>0,
\end{aligned}
$$

where $k, \theta, \sigma$ and $\lambda$ are positive constants and satisfy the condition: $2 k \theta>\sigma^{2}$. It ensures $L(t)>0$ for $\forall t \in[0, T]$.

The initial wealth of investors at time $t=0$ is denoted by $x_{0}>0$. Denote the wealth process at time $t$ by $X(t)$ and the amount in the stock by $\pi(t)$, then $X(t)-\pi(t)$ is the amount in the risk-free asset. Let $C(t)$ be the consumption amount at time $t$. Therefore, the wealth process $X(t)$ under the investment-consumption strategy $(\pi(t), C(t))$ is given by

$$
X(t)=(r X(t)+\pi(t) \lambda L(t)-C(t)) \mathrm{d} t+\pi(t) \sqrt{L(t)} \mathrm{d} W_{1}(t), \quad X(0)=x_{0}>0 .
$$

Definition 1 (admissible strategy) An investment-consumption policy $(\pi(t), C(t))$ is said to be admissible if $\pi(t)$ satisfies the following conditions:

(i) $(\pi(t), C(t))$ is $\mathscr{F}_{t}$-progressively measurable and satisfies $\int_{0}^{T} \pi^{2}(t) \mathrm{d} t<\infty$ and $\int_{0}^{T} C(t) \mathrm{d} t<$ $\infty$;

(ii) $E\left(\int_{0}^{T} \pi^{2}(t) L(t) \mathrm{d} t\right)<\infty$;

(iii) Equation (4) has a unique pathwise solution under any $\pi(t)$.

We denote the set of all admissible strategies by $\Gamma$. The aim of investors is to maximize the expected discount utility of intermediate consumption and terminal wealth, i.e.,

$$
\underset{(\pi(t), C(t)) \in \Gamma}{\operatorname{Maximize}} E\left(\alpha \int_{0}^{T} \mathrm{e}^{-\beta t} U_{1}(C(t)) \mathrm{d} t+(1-\alpha) \mathrm{e}^{-\beta T} U_{2}(X(T))\right),
$$

where $U(x)$ is utility function, which satisfies the conditions: first-order derivative $\dot{U}(x)>0$ and two-order derivative $\ddot{U}(x)<0$.

In this paper, we assume that the risky preference of investors satisfies hyperbolic absolute risk aversion (HARA) utility function. In the utility theory, HARA utility with parameters $\eta, p$ 
and $q$ is given by

$$
U_{1}(x)=U_{2}(x)=U(\eta, p, q, x)=\frac{1-p}{q p}\left(\frac{q}{1-p} x+\eta\right)^{p}, \quad q>0, \quad p<1, \quad p \neq 0 .
$$

In reality, HARA utility recovers power utility, logarithm utility and exponential utility as special cases.

(i) If we choose $\eta=0$ and $q=1-p$, then we have

$$
U(0, p, 1-p, x)=\frac{x^{p}}{p} \triangleq U_{\text {pow }}(x) .
$$

(ii) If we choose $\eta=0, p \rightarrow 0$ and $q \rightarrow 1$, then we have

$$
U(0, p, q, x)=\ln x \triangleq U_{\log }(x) .
$$

(iii) If we choose $\eta=1$ and $p \rightarrow-\infty$, then we have

$$
U(1, p, q, x)=-\frac{\mathrm{e}^{-q x}}{q} \triangleq U_{\exp }(x) .
$$

\section{HJB Equation and Legendre Transform}

We define the value function $H(t, l, x)$ as

$H(t, l, x)=\operatorname{Max}_{(\pi(t), C(t)) \in \Gamma} E\left(\alpha \int_{0}^{T} \mathrm{e}^{-\beta t} U_{1}(C(t)) \mathrm{d} t+(1-\alpha) \mathrm{e}^{-\beta T} U_{2}(X(T)) \mid X(t)=x, L(t)=l\right)$,

with boundary condition $H(T, l, x)=(1-\alpha) \mathrm{e}^{-\beta T} U_{2}(x)$.

According to stochastic dynamic programming principle, $H(t, l, x)$ is a continuous solution to the following Hamilton-Jacobi-Bellman (HJB) equation

$$
\begin{gathered}
\underset{(\pi(t), C(t)) \in \Gamma}{\operatorname{Max}}\left\{H_{t}+(r x+\pi(t) \lambda l-C(t)) H_{x}+\frac{1}{2} \pi^{2}(t) l H_{x x}+k(\theta-l) H_{l}\right. \\
+ \\
\left.+\frac{1}{2} \sigma^{2} l H_{l l}+\pi(t) \sigma l H_{x l}+\alpha \mathrm{e}^{-\beta t} U_{1}(C(t))\right\}=0,
\end{gathered}
$$

where $H_{t}, H_{x}, H_{x x}, H_{l}, H_{l l}$ and $H_{x l}$ are first-order and second-order derivatives of $H(t, l, x)$ with respect to the variables $t, x, l$.

The necessary condition of the optimality principle helps us to obtain

$$
\pi^{*}(t)=-\lambda \frac{H_{x}}{H_{x x}}-\sigma \frac{H_{x l}}{H_{x x}}, \quad \dot{U}_{1}\left(C^{*}(t)\right)=\frac{H_{x}}{\alpha \mathrm{e}^{-\beta t}} .
$$

Substituting (7) back into (6), we get

$$
\begin{aligned}
& H_{t}+r x H_{x}+k(\theta-l) H_{l}+\frac{1}{2} \sigma^{2} l H_{l l}-\frac{1}{2} \lambda^{2} l \frac{H_{x}^{2}}{H_{x x}}-\sigma \lambda l \frac{H_{x} H_{x l}}{H_{x x}} \\
& -\frac{1}{2} \sigma^{2} l \frac{H_{x l}^{2}}{H_{x x}}-C^{*}(t) H_{x}+\alpha \mathrm{e}^{-\beta t} U_{1}\left(C^{*}(t)\right)=0 .
\end{aligned}
$$

with boundary condition $H(T, l, x)=(1-\alpha) \mathrm{e}^{-\beta T} \frac{1-p}{q p}\left(\frac{q}{1-p} x+\eta\right)^{p}$. 
Due to the complexity of boundary condition, we find no way to accurately conjecture the structure of solution to the equation (8). Therefore, we introduce the following Legendre transform-dual theory.

Definition 2. Let $f: R^{n} \rightarrow R$ be a convex function. Legendre transform can be defined as follows:

$$
L(z)=\max _{x}\{f(x)-z x\},
$$

then $L(z)$ is called Legendre dual function of $f(x)$ (cf. Chang, et al. ${ }^{[11]}$ and Jonsson and Sircar $\left.{ }^{[12]}\right)$.

If $f(x)$ is strictly convex, the maximum in the equation (9) will be attained at just one point, which we denote by $\tilde{x}_{0}$. We can attain at the unique solution by the first-order condition:

$$
\dot{f}(x)-z=0 .
$$

So we have

$$
L(z)=f\left(\tilde{x}_{0}\right)-z \tilde{x}_{0}
$$

Following Chang, et al. ${ }^{[11]}$ and Jonsson and Sircar ${ }^{[12]}$, Legendre transform can be defined by

$$
\hat{H}(t, l, z)=\sup _{x>0}\{H(t, l, x)-z x\}
$$

where $z>0$ denotes the dual variable to $x$. The value of $x$ where this optimum is attained is denoted by $g(t, l, z)$, so we have

$$
g(t, l, z)=\inf _{x>0}\{x \mid H(t, l, x) \geqslant z x+\hat{H}(t, l, z)\} .
$$

The relationship between $\hat{H}(t, l, z)$ and $g(t, l, z)$ is given by

$$
g(t, l, z)=-\hat{H}_{z}(t, l, z)
$$

Hence, we can choose either one of the two functions $g(t, l, z)$ and $\hat{H}(t, l, z)$ as the dual function of $H(t, l, x)$. Here, we choose $g(t, l, z)$. Moreover, we have

$$
H_{x}=z, \quad \hat{H}(t, l, z)=H(t, l, g)-z g, \quad g(t, l, z)=x .
$$

Differentiating (13) with respect to $t, l$ and $x$, we get

$$
H_{t}=\hat{H}_{t}, \quad H_{x}=z, \quad H_{x x}=-\frac{1}{\hat{H}_{z z}}, \quad H_{l}=\hat{H}_{l}, \quad H_{l l}=\hat{H}_{l l}-\frac{\hat{H}_{l z}^{2}}{\hat{H}_{z z}}, \quad H_{x l}=-\frac{\hat{H}_{l z}}{\hat{H}_{z z}} .
$$

Notice that $H(T, l, x)=(1-\alpha) \mathrm{e}^{-\beta T} U_{2}(x)$, then at the terminal time $T$, we can define

$$
\hat{H}(T, l, z)=\sup _{x>0}\{H(T, l, x)-z x\}, \quad g(T, l, z)=\inf _{x>0}\{x \mid H(T, l, x) \geqslant z x+\hat{H}(T, l, z)\} .
$$

So we have $g(T, l, z)=\left(\dot{U}_{2}\right)^{-1}\left(\frac{z}{(1-\alpha) \mathrm{e}^{-\beta T}}\right)$, where $\left(\dot{U}_{2}\right)^{-1}(\cdot)$ is taken as the inverse of marginal utility.

Putting (14) in the equation (8), we get

$$
\hat{H}_{t}+r z g+k(\theta-l) \hat{H}_{l}+\frac{1}{2} \sigma^{2} l \hat{H}_{l l}+\frac{1}{2} \lambda^{2} l z^{2} \hat{H}_{z z}-\sigma \lambda l z \hat{H}_{l z}-C^{*}(t) z+\alpha \mathrm{e}^{-\beta t} U_{1}\left(C^{*}(t)\right)=0 .
$$


Differentiating (15) with respect to $z$ and applying (12), we derive

$$
\begin{aligned}
& g_{t}-r g+\left(\lambda^{2} l-r\right) z g_{z}+\frac{1}{2} \lambda^{2} l z^{2} g_{z z}+(k \theta-k l-\sigma \lambda l) g_{i}+\frac{1}{2} l \sigma^{2} g_{l l} \\
& -\sigma \lambda l z g_{l z}+\frac{\partial\left(C^{*}(t) z\right)}{\partial z}-\frac{\partial\left(\alpha \mathrm{e}^{-\beta t} U_{1}\left(C^{*}(t)\right)\right)}{\partial z}=0,
\end{aligned}
$$

with terminal condition

$$
g(T, l, z)=\frac{1-p}{q}(1-\alpha)^{-\frac{1}{p-1}}\left(\mathrm{e}^{\beta T}\right)^{\frac{1}{p-1}} z^{\frac{1}{p-1}}-\frac{1-p}{q} \eta .
$$

\section{Optimal Consumption and Portfolio Decision}

We conjecture the structure of solution to (16) with the following form

$$
g(t, l, z)=\frac{1-p}{q}(1-\alpha)^{-\frac{1}{p-1}}\left(\mathrm{e}^{\beta t}\right)^{\frac{1}{p-1}} z^{\frac{1}{p-1}} f(t, l)-\frac{1-p}{q} \eta h(t), \quad f(T, l)=1, \quad h(T)=1 .
$$

The partial derivatives of $g(t, l, z)$ are get as follows:

$$
\begin{aligned}
& g_{t}=\frac{1-p}{q}(1-\alpha)^{-\frac{1}{p-1}}\left(\mathrm{e}^{\beta t}\right)^{\frac{1}{p-1}} z^{\frac{1}{p-1}}\left(f_{t}+\frac{1}{p-1} \beta f\right)-\frac{1-p}{q} \eta \dot{h}(t), \\
& g_{z}=\frac{1-p}{q}(1-\alpha)^{-\frac{1}{p-1}}\left(\mathrm{e}^{\beta t}\right)^{\frac{1}{p-1}} z^{\frac{1}{p-1}-1} f \frac{1}{p-1}, \\
& g_{z z}=\frac{1-p}{q}(1-\alpha)^{-\frac{1}{p-1}}\left(\mathrm{e}^{\beta t}\right)^{\frac{1}{p-1}} z^{\frac{1}{p-1}-2} f \frac{2-p}{(p-1)^{2}}, \\
& g_{l}=\frac{1-p}{q}(1-\alpha)^{-\frac{1}{p-1}}\left(\mathrm{e}^{\beta t}\right)^{\frac{1}{p-1}} z^{\frac{1}{p-1}} f_{l}, \quad g_{l l}=\frac{1-p}{q}(1-\alpha)^{-\frac{1}{p-1}}\left(\mathrm{e}^{\beta t}\right)^{\frac{1}{p-1}} z^{\frac{1}{p-1}} f_{l l}, \\
& g_{l z}=\frac{1-p}{q}(1-\alpha)^{-\frac{1}{p-1}}\left(\mathrm{e}^{\beta t}\right)^{\frac{1}{p-1}} z^{\frac{1}{p-1}-1} f_{l} \frac{1}{p-1} .
\end{aligned}
$$

According to (7), we get

$$
C^{*}(t)=\frac{1-p}{q} \alpha^{-\frac{1}{p-1}}\left(\mathrm{e}^{\beta t}\right)^{\frac{1}{p-1}} z^{\frac{1}{p-1}}-\frac{1-p}{q} \eta .
$$

Substituting (17) and (18) back into (16), we get

$$
\begin{aligned}
& \frac{1-p}{q}(1-\alpha)^{-\frac{1}{p-1}}\left(\mathrm{e}^{\beta t}\right)^{\frac{1}{p-1}} z^{\frac{1}{p-1}}\left(f_{t}+\left(\frac{1}{p-1} \beta-\frac{p}{p-1} r\right) f+\frac{p}{2(p-1)^{2}} \lambda^{2} l f\right. \\
& \left.-\left(k+\frac{p}{p-1} \sigma \lambda\right) l f_{l}+k \theta f_{l}+\frac{1}{2} \sigma^{2} l f_{l l}+\left(\frac{\alpha}{1-\alpha}\right)^{-\frac{1}{p-1}}\right) \\
& -\frac{1-p}{q} \eta(\dot{h}(t)-r h(t)+1)=0 .
\end{aligned}
$$

Eliminating the dependence on $z$ and $\eta$, we have

$$
\begin{aligned}
& \dot{h}(t)-r h(t)+1=0, \quad h(T)=1 . \\
& f_{t}+\left(\frac{1}{p-1} \beta-\frac{p}{p-1} r\right) f+\frac{p}{2(p-1)^{2}} \lambda^{2} l f-\left(k+\frac{p}{p-1} \sigma \lambda\right) l f_{l} \\
& +k \theta f_{l}+\frac{1}{2} \sigma^{2} l f_{l l}+\left(\frac{\alpha}{1-\alpha}\right)^{-\frac{1}{p-1}}=0, \quad f(T, l)=1 .
\end{aligned}
$$


The solution to (19) is given by

$$
h(t)=\mathrm{e}^{-r(T-t)}+\frac{1}{r}\left(1-\mathrm{e}^{-r(T-t)}\right) .
$$

The solution to (20) is described as the following Lemma 1 and Lemma 2.

Lemma 1 Given that $f(t, l)=\left(\frac{\alpha}{1-\alpha}\right)^{-\frac{1}{p-1}} \int_{t}^{T} \hat{f}(s, l) \mathrm{d} s+\hat{f}(t, l)$ is the solution to $(20)$, then $\hat{f}(t, l)$ satisfies

$$
\begin{aligned}
& \hat{f}_{t}+\left(\frac{1}{p-1} \beta-\frac{p}{p-1} r\right) \hat{f}+\frac{p}{2(p-1)^{2}} \lambda^{2} l \hat{f}-\left(k+\frac{p}{p-1} \sigma \lambda\right) l \hat{f}_{l} \\
& +k \theta \hat{f}_{l}+\frac{1}{2} \sigma^{2} l \hat{f}_{l l}=0, \quad \hat{f}(T, l)=1 .
\end{aligned}
$$

Proof Introducing the following variational operator

$$
\nabla f(t, l)=\left(\frac{1}{p-1} \beta-\frac{p}{p-1} r\right) f+\frac{p}{2(p-1)^{2}} \lambda^{2} l f-\left(k+\frac{p}{p-1} \sigma \lambda\right) l f_{l}+k \theta f_{l}+\frac{1}{2} \sigma^{2} l f_{l l},
$$

we can rewrite $(20)$ as

$$
\frac{\partial f(t, l)}{\partial t}+\nabla f(t, l)+\left(\frac{\alpha}{1-\alpha}\right)^{-\frac{1}{p-1}}=0, \quad f(T, l)=1
$$

According to $f(t, l)=\left(\frac{\alpha}{1-\alpha}\right)^{-\frac{1}{p-1}} \int_{t}^{T} \hat{f}(s, l) \mathrm{d} s+\hat{f}(t, l)$, we find

$$
\begin{aligned}
\frac{\partial f(t, l)}{\partial t} & =-\left(\frac{\alpha}{1-\alpha}\right)^{-\frac{1}{p-1}} \hat{f}(t, l)+\frac{\partial \hat{f}(t, l)}{\partial t} \\
& =\left(\frac{\alpha}{1-\alpha}\right)^{-\frac{1}{p-1}}\left(\int_{t}^{T} \frac{\partial \hat{f}(s, l)}{\partial s} \mathrm{~d} s-\hat{f}(T, l)\right)+\frac{\partial \hat{f}(t, l)}{\partial t} . \\
\nabla f(t, l) & =\left(\frac{\alpha}{1-\alpha}\right)^{-\frac{1}{p-1}} \int_{t}^{T} \nabla \hat{f}(s, l) \mathrm{d} s+\nabla \hat{f}(t, l) . \\
f(T, l)=1 & \Rightarrow \hat{f}(T, l)=1 .
\end{aligned}
$$

Substituting (25) and (26) back into (24), we get

$$
\left(\frac{\alpha}{1-\alpha}\right)^{-\frac{1}{p-1}}\left(\int_{t}^{T}\left(\frac{\partial \hat{f}(s, l)}{\partial s}+\nabla \hat{f}(s, l)\right) \mathrm{d} s-\hat{f}(T, l)+1\right)+\frac{\partial \hat{f}(t, l)}{\partial t}+\nabla \hat{f}(t, l)=0 .
$$

Using $\hat{f}(T, l)=1$, we have

$$
\frac{\partial \hat{f}(t, l)}{\partial t}+\nabla \hat{f}(t, l)=0, \quad \hat{f}(T, l)=1
$$

Therefore, (22) is verified.

Lemma 2 Given that the solution to $(22)$ is of the structure $\hat{f}(t, l)=\mathrm{e}^{D_{1}(t)+D_{2}(t) l}$, with boundary conditions $D_{1}(T)=0$ and $D_{2}(T)=0$, we have the following conclusions:

(i) If $p<\frac{k^{2}}{(k+\sigma \lambda)^{2}}$ and $p \neq 0, D_{2}(t)$ and $D_{1}(t)$ are given by (35) and (36) respectively. 
(ii) If $p=\frac{k^{2}}{(k+\sigma \lambda)^{2}}$ and $p \neq 0, D_{2}(t)$ and $D_{1}(t)$ are given by (37) and (38) respectively.

(iii) If $\frac{k^{2}}{(k+\sigma \lambda)^{2}}<p<1, D_{2}(t)$ and $D_{1}(t)$ are given by (39) and (40) respectively.

Proof Putting $\hat{f}(t, l)=\mathrm{e}^{D_{1}(t)+D_{2}(t) l}$ into (22) and separating the variables, we get

$$
\begin{aligned}
& \mathrm{e}^{D_{1}(t)+D_{2}(t) l}\left(\dot{D}_{1}(t)+\frac{\beta}{p-1}-\frac{p}{p-1} r+k \theta D_{2}(t)\right. \\
& \left.+\left(\dot{D}_{2}(t)+\frac{p}{2(p-1)^{2}} \lambda^{2}-\left(k+\frac{p}{p-1} \sigma \lambda\right) D_{2}(t)+\frac{1}{2} \sigma^{2} D_{2}^{2}(t)\right) l\right)=0 .
\end{aligned}
$$

Eliminating the dependence on $l$, we obtain the following ordinary differential equations:

$$
\begin{gathered}
\dot{D}_{2}(t)+\frac{p}{2(p-1)^{2}} \lambda^{2}-\left(k+\frac{p}{p-1} \sigma \lambda\right) D_{2}(t)+\frac{1}{2} \sigma^{2} D_{2}^{2}(t)=0, \quad D_{2}(T)=0 . \\
\dot{D}_{1}(t)+\frac{\beta}{p-1}-\frac{p}{p-1} r+k \theta D_{2}(t)=0, \quad D_{1}(T)=0 .
\end{gathered}
$$

We rewrite (31) and (32) as follows.

$$
\begin{aligned}
& \dot{D}_{2}(t)=-\frac{1}{2} \sigma^{2} D_{2}^{2}(t)+\left(k+\frac{p}{p-1} \sigma \lambda\right) D_{2}(t)-\frac{p}{2(p-1)^{2}} \lambda^{2} . \\
& D_{1}(t)=\left(\frac{\beta}{p-1}-\frac{p}{p-1} r\right)(T-t)+k \theta \int_{t}^{T} D_{2}(s) \mathrm{d} s .
\end{aligned}
$$

For the equation (33), we find that the solution to (33) depends on the number of the root of quadratic equation

$$
-\frac{1}{2} \sigma^{2} D_{2}^{2}(t)+\left(k+\frac{p}{p-1} \sigma \lambda\right) D_{2}(t)-\frac{p}{2(p-1)^{2}} \lambda^{2}=0 .
$$

We discuss and solve (33) and (34) as follows.

(i) If $p<\frac{k^{2}}{(k+\sigma \lambda)^{2}}$ and $p \neq 0$, we have

$$
\begin{aligned}
D_{2}(t)= & \frac{m_{1} m_{2}\left(1-\exp \left\{-\frac{1}{2} \sigma^{2}\left(m_{1}-m_{2}\right)(T-t)\right\}\right)}{m_{1}-m_{2} \cdot \exp \left\{-\frac{1}{2} \sigma^{2}\left(m_{1}-m_{2}\right)(T-t)\right\}}, \\
D_{1}(t)= & \left(\frac{\beta}{p-1}-\frac{p}{p-1} r+k \theta m_{2}\right)(T-t) \\
& +\frac{2 k \theta}{\sigma^{2}} \ln \frac{m_{1}-m_{2}}{m_{1}-m_{2} \cdot \exp \left\{-\frac{1}{2} \sigma^{2}\left(m_{1}-m_{2}\right)(T-t)\right\}},
\end{aligned}
$$

where

$$
m_{1,2}=\frac{(p-1) k+p \sigma \lambda}{(p-1) \sigma^{2}} \pm \frac{\sqrt{\Delta_{1}}}{\sigma^{2}}, \quad \Delta_{1}=\frac{1}{p-1}\left(p(k+\sigma \lambda)^{2}-k^{2}\right) .
$$

(ii) If $p=\frac{k^{2}}{(k+\sigma \lambda)^{2}}$ and $p \neq 0$, we get

$$
\begin{aligned}
D_{2}(t) & =\frac{\sigma^{2} m_{3}^{2}(T-t)}{\sigma^{2} m_{3}(T-t)+2} \\
D_{1}(t) & =\left(\frac{\beta}{p-1}-\frac{p}{p-1} r+k \theta m_{3}\right)(T-t)+\frac{2 k \theta}{\sigma^{2}} \ln \frac{2}{\sigma^{2} m_{3}(T-t)+2},
\end{aligned}
$$


where

$$
m_{3}=\frac{(p-1) k+p \sigma \lambda}{(p-1) \sigma^{2}}
$$

(iii) If $\frac{k^{2}}{(k+\sigma \lambda)^{2}}<p<1$, we obtain

$$
\begin{aligned}
D_{2}(t)= & m_{3}-\frac{\sqrt{-\Delta_{1}}}{\sigma^{2}} \tan \left(\arctan \frac{\sigma^{2} m_{3}}{\sqrt{-\Delta_{1}}}-\frac{\sqrt{-\Delta_{1}}}{2}(T-t)\right) . \\
D_{1}(t)= & \left(\frac{\beta}{p-1}-\frac{p}{p-1} r+k \theta m_{3}\right)(T-t)+\frac{2 k \theta}{\sigma^{2}} \ln \cos \left(\arctan \frac{\sigma^{2} m_{3}}{\sqrt{-\Delta_{1}}}\right) \\
& -\frac{2 k \theta}{\sigma^{2}} \ln \cos \left(\arctan \frac{\sigma^{2} m_{3}}{\sqrt{-\Delta_{1}}}-\frac{\sqrt{-\Delta_{1}}}{2}(T-t)\right) .
\end{aligned}
$$

In short, Lemma 2 is proved.

In addition, according to (7), (13), (14), (17) and (18), we derive

$$
\begin{aligned}
\frac{H_{x}}{H_{x x}} & =-z \hat{H}_{z z}=z g_{z}=\frac{1-p}{q}(1-\alpha)^{-\frac{1}{p-1}}\left(\mathrm{e}^{\beta t}\right)^{\frac{1}{p-1}} z^{\frac{1}{p-1}} f \frac{1}{p-1} \\
& =\frac{1}{p-1}\left(g+\frac{1-p}{q} \eta h(t)\right)=-\frac{1}{1-p}\left(x+\frac{1-p}{q} \eta h(t)\right) . \\
\frac{H_{x l}}{H_{x x}} & =\hat{H}_{l z}=-g_{l}=-\frac{1-p}{q}(1-\alpha)^{-\frac{1}{p-1}}\left(\mathrm{e}^{\beta t}\right)^{\frac{1}{p-1}} z^{\frac{1}{p-1}} f_{l} \\
& =-\left(g+\frac{1-p}{q} \eta h(t)\right) \frac{f_{l}}{f}=-\left(x+\frac{1-p}{q} \eta h(t)\right) \frac{f_{l}}{f} . \\
C^{*}(t) & =\left(\frac{\alpha}{1-\alpha}\right)^{-\frac{1}{p-1}}\left(g+\frac{1-p}{q} \eta h(t)\right) f^{-1}-\frac{1-p}{q} \eta \\
& =\left(\frac{\alpha}{1-\alpha}\right)^{-\frac{1}{p-1}}\left(x+\frac{1-p}{q} \eta h(t)\right) f^{-1}-\frac{1-p}{q} \eta .
\end{aligned}
$$

Further, by using $g(t, l, z)=x$, we obtain

$$
z=(1-\alpha) \mathrm{e}^{-\beta t}\left(\frac{q}{1-p} x+\eta h(t)\right)^{p-1} f^{1-p}(t, l) .
$$

Using $H_{x}=z$ and integrating both sides, we get

$$
H_{H A R A}^{*}(t, l, x)=(1-\alpha) \mathrm{e}^{-\beta t} \frac{1-p}{q p}\left(\frac{q}{1-p} x+\eta h(t)\right)^{p} f^{1-p}(t, l) .
$$

Summarizing what are mentioned above, we have the following conclusions.

Theorem 1 Under HARA utility criterion

$$
U_{1}(x)=U_{2}(x)=U(\eta, p, q, x)=\frac{1-p}{q p}\left(\frac{q}{1-p} x+\eta\right)^{p}, \quad q>0, p<1, p \neq 0,
$$

the optimal consumption-investment strategies of the problem (5) are given by

$$
\pi_{H A R A}^{*}(t)=\frac{\lambda}{1-p}\left(X(t)+\frac{1-p}{q} \eta h(t)\right)+\sigma\left(X(t)+\frac{1-p}{q} \eta h(t)\right) \frac{f_{l}}{f(t, l)},
$$




$$
C_{H A R A}^{*}(t)=\left(\frac{\alpha}{1-\alpha}\right)^{-\frac{1}{p-1}}\left(X(t)+\frac{1-p}{q} \eta h(t)\right) f^{-1}(t, l)-\frac{1-p}{q} \eta,
$$

with the optimal value function given by (44), where $h(t)$ is given by (21), and

$$
\begin{aligned}
& f(t, l)=\left(\frac{\alpha}{1-\alpha}\right)^{-\frac{1}{p-1}} \int_{t}^{T} \mathrm{e}^{D_{1}(s)+D_{2}(s) l} \mathrm{~d} s+\mathrm{e}^{D_{1}(t)+D_{2}(t) l}, \\
& f_{l}=\frac{\partial f(t, l)}{\partial l}=\left(\frac{\alpha}{1-\alpha}\right)^{-\frac{1}{p-1}} \int_{t}^{T} D_{2}(s) \mathrm{e}^{D_{1}(s)+D_{2}(s) l} \mathrm{~d} s+D_{2}(t) \mathrm{e}^{D_{1}(t)+D_{2}(t) l} .
\end{aligned}
$$

Here, $D_{2}(t)$ and $D_{1}(t)$ are determined by Lemma 2.

Compared with the existing literatures, the conclusions from Theorem 1 is of generality. In addition, they consist of the following three main special cases.

Corollary 1 If utility function is given by $U_{\text {pow }}(x)=\frac{x^{p}}{p}$, the optimal consumptioninvestment strategies of the problem (5) are given by

$$
\begin{gathered}
\pi_{\text {pow }}^{*}(t)=\frac{\lambda}{1-p} X(t)+\sigma \frac{f_{l}}{f(t, l)} X(t), \\
C_{\text {pow }}^{*}(t)=\left(\frac{\alpha}{1-\alpha}\right)^{-\frac{1}{p-1}} f^{-1}(t, l) X(t),
\end{gathered}
$$

with the optimal value function given by

$$
H_{\text {pow }}^{*}(t, l, x)=(1-\alpha) \mathrm{e}^{-\beta t} \frac{1}{p} x^{p} f^{1-p}(t, l),
$$

where $f(t, l)$ and $f_{l}$ are given by (47) and (48) respectively.

Proof If $\eta=0$ and $q=1-p$, we know that HARA utility is reduced to power utility. Putting $\eta=0$ and $q=1-p$ into Theorem 1, it is obvious that (49) (51) hold.

Corollary 2 If utility function is given by $U_{\log }(x)=\ln x$, the optimal consumptioninvestment strategies of the problem (5) are given by

$$
\pi_{\log }^{*}(t)=\lambda X(t), \quad C_{\log }^{*}(t)=\frac{\alpha}{1-\alpha} f^{-1}(t) X(t),
$$

with the optimal value function given by

$$
H_{\log }^{*}(t, l, x)=(1-\alpha) \mathrm{e}^{-\beta t} f(t) \ln x,
$$

where $f(t)$ is given by (54).

Proof If $\eta=0, p \rightarrow 0$ and $q \rightarrow 1$ in the HARA utility, we get logarithmic utility $U_{\log }(x)=$ $\ln x$. Under this situation, we have $D_{2}(t)=0$ and $D_{1}(t)=-\beta(T-t)$. In addition, according to Lemma 1 and Lemma 2, we get

$$
f(t, l)=\left(\frac{\alpha}{1-\alpha}\right) \frac{1}{\beta}\left(1-\mathrm{e}^{-\beta(T-t)}\right)+\mathrm{e}^{-\beta(T-t)} \triangleq f(t) .
$$

Therefore, Corollary 2 holds.

Corollary 3 If utility function is given by $U_{\exp }(x)=-\frac{\mathrm{e}^{-q x}}{q}$, the optimal consumptioninvestment strategies of the problem (5) are given by 


$$
\begin{aligned}
\pi_{\exp }^{*}(t)=\frac{1}{q} \lambda h(t)+\frac{1}{q} \sigma\left(\int_{t}^{T} \mathrm{e}^{-r(T-s)} \varphi_{2}(s)\left(\varphi_{1}(s)+\varphi_{2}(s) l\right) \mathrm{d} s\right. \\
\left.+\mathrm{e}^{-r(T-t)} \varphi_{2}(t)\left(\varphi_{1}(t)+\varphi_{2}(t) l\right)\right), \\
C_{\exp }^{*}(t)=-\frac{1}{q}\left\{\ln \frac{1-\alpha}{\alpha}-q h^{-1}(t) X(t)+h^{-1}(t)\left(\ln \frac{\alpha}{1-\alpha} \cdot \int_{t}^{T} \mathrm{e}^{-r(T-s)} \mathrm{d} s\right.\right. \\
\left.\left.\quad+\int_{t}^{T} \mathrm{e}^{-r(T-s)}\left(\varphi_{1}(s)+\varphi_{2}(s) l\right) \mathrm{d} s+\mathrm{e}^{-r(T-t)}\left(\varphi_{1}(t)+\varphi_{2}(t) l\right)\right)\right\},
\end{aligned}
$$

with the optimal value function given by (63), where $h(t)$ is given by (21), and $\varphi_{1}(t)$ and $\varphi_{2}(t)$ are given by (61) and (62) respectively.

Proof If $\eta=1$ and $p \rightarrow-\infty$, we find that HARA utility is degenerated to exponential utility.

Further, we derive that

$$
D_{2}(t) \rightarrow 0, \quad D_{1}(t) \rightarrow-r(T-t), \quad f(t, l) \rightarrow h(t), \quad f_{l} \rightarrow 0 .
$$

So (44) can be rewritten as

$$
\begin{aligned}
H_{H A R A}^{*}(t, l, x) & =(1-\alpha) \mathrm{e}^{-\beta t} \frac{1-p}{q p}\left(\frac{q}{1-p} x+\eta h(t)\right)^{p} f^{1-p}(t, l) \\
& =(1-\alpha) \mathrm{e}^{-\beta t} \frac{1-p}{q p}\left(1+\frac{q}{1-p} x h^{-1}(t)\right)^{p} f(t, l)\left(f^{-1}(t, l) h(t)\right)^{p} .
\end{aligned}
$$

Taking the limitation $p \rightarrow-\infty$, we have

$$
\begin{aligned}
H_{\exp }^{*}(t, l, x) & =\lim _{p \rightarrow-\infty} H_{H A R A}^{*}(t, l, x) \\
& =-(1-\alpha) \mathrm{e}^{-\beta t} \cdot \frac{1}{q} \mathrm{e}^{-q x h^{-1}(t)} \cdot \lim _{p \rightarrow-\infty} f(t, l) \cdot \lim _{p \rightarrow-\infty} \mathrm{e}^{\ln \left(f^{-1}(t, l) h(t)\right)^{p}} .
\end{aligned}
$$

On the other hand, we derive

$$
\begin{aligned}
\lim _{p \rightarrow-\infty} \ln \left(f^{-1}(t, l) h(t)\right)^{p}=\lim _{p \rightarrow-\infty} \frac{\ln \left(f^{-1}(t, l) h(t)\right)}{1 / p} \\
=h^{-1}(t)\left(\ln \frac{\alpha}{1-\alpha} \cdot \int_{t}^{T} \mathrm{e}^{-r(T-s)} \mathrm{d} s+\int_{t}^{T} \mathrm{e}^{-r(T-s)}\left(\varphi_{1}(s)+\varphi_{2}(s) l\right) \mathrm{d} s\right. \\
\left.\quad+\mathrm{e}^{-r(T-t)}\left(\varphi_{1}(t)+\varphi_{2}(t) l\right)\right)
\end{aligned}
$$

where

$$
\begin{aligned}
\varphi_{1}(t) & =(r-\beta)(T-t)+\frac{k \theta \lambda}{\sigma}(T-t)-\frac{k \theta}{\sigma^{2}}\left(1-\mathrm{e}^{\sigma \lambda(T-t)}\right), \\
\varphi_{2}(t) & =\frac{\lambda}{\sigma}\left(1-\mathrm{e}^{\sigma \lambda(T-t)}\right) .
\end{aligned}
$$


As a matter of fact, the second limitation in the equation (60) is a $\frac{0}{0}$. By using the L'Hôpital's rule, we can obtain $(60)$.

Therefore, putting (60) in (59), we get

$$
\begin{aligned}
& H_{\exp }^{*}(t, l, x) \\
= & -(1-\alpha) \mathrm{e}^{-\beta t} \cdot h(t) \cdot \frac{1}{q} \exp \left\{-q x h^{-1}(t)+h^{-1}(t)\left(\ln \frac{\alpha}{1-\alpha} \cdot \int_{t}^{T} \mathrm{e}^{-r(T-s)} \mathrm{d} s\right.\right. \\
& \left.\left.+\int_{t}^{T} \mathrm{e}^{-r(T-s)}\left(\varphi_{1}(s)+\varphi_{2}(s) l\right) \mathrm{d} s+\mathrm{e}^{-r(T-t)}\left(\varphi_{1}(t)+\varphi_{2}(t) l\right)\right)\right\} .
\end{aligned}
$$

In addition, we derive

$$
\begin{aligned}
\frac{H_{x}}{H_{x x}} & =-\frac{1}{q} h(t), \\
\frac{H_{x l}}{H_{x x}} & =-\frac{1}{q}\left(\int_{t}^{T} \mathrm{e}^{-r(T-s)} \varphi_{2}(s)\left(\varphi_{1}(s)+\varphi_{2}(s) l\right) \mathrm{d} s+\mathrm{e}^{-r(T-t)} \varphi_{2}(t)\left(\varphi_{1}(t)+\varphi_{2}(t) l\right)\right) .
\end{aligned}
$$

Putting (64) and (65) into (7), we get (55) and (56).

\section{Conclusions}

HARA utility is a general utility function in the utility theory and is seldom studied in recent decade years because of its complicated structure. In this paper, Legendre transform-dual technique and stochastic dynamic programming principle are presented to deal with the optimal consumption-investment strategy with Heston's stochastic volatility model under HARA utility criterion. The closed-form solution to the optimal consumption-investment strategy is successfully obtained. Our research result displays that Legendre transform-dual theory along with stochastic dynamic programming principle is an effective methodology in dealing with dynamic portfolio selection problems under HARA utility.

\section{References}

[1] Merton R C. Lifetime portfolio selection under uncertainty: The continuous-time case. The Review of Economics and Statistics, 1969, 51(3): 247-257.

[2] Merton R C. Optimum consumption and portfolio rules in a continuous-time model. Journal of Economic Theory, 1971, 3(4): 373-413.

[3] Fleming W H, Hernandez-hernandez D. An optimal consumption model with stochastic volatility. Finance and Stochastics, 2003, 7(2): 245-262.

[4] Chacko G, Viceira L M. Dynamic consumption and portfolio choice with stochastic volatility in incomplete markets. Review of Financial Studies, 2005, 18(4): 1369-1402.

[5] Ekeland I, Pirvu T A. Investment and consumption without commitment. Mathematics and Financial Economics, 2008, 2(1): 57-86.

[6] Pirvu T A, Zhang H. Investment-consumption with regime-switching discount rates. Mathematical Social Sciences, 2014, 71(5): 142-150.

[7] Zhao Q, Shen Y, Wei J Q. Consumption-investment strategies with non-exponential discounting and logarithmic utility. European Journal of Operational Research, 2014, 238: 824-835.

[8] Zou Z R, Chen S, Wedge L. Finite horizon consumption and portfolio decisions with stochastic hyperbolic discounting. Journal of Mathematical Economics, 2014, 52(1): 70-80. 
[9] Jung E J, Kim J H. Optimal investment strategies for the HARA utility under the constant elasticity of variance model. Insurance: Mathematics and Economics, 2012, 51(1): 667-673.

[10] Chang H, Rong X M. Legendre transform-dual solution for a class of investment and consumption problems with HARA utility.Mathematical Problems in Engineering, 2014, Article ID 656438, 7 pages, http: //dx.doi.org /10.1155/2014/656438.

[11] Chang H, Chang K, Lu J M. Portfolio selection with liability and affine interest rate in the HARA utility framework. Abstract and Applied Analysis, 2014, Article ID 312640, 12 pages, http: //dx.doi.org $/ 10.1155 / 2014 / 312640$.

[12] Jonsson M, Sircar R. Optimal investment problems and volatility homogenization approximations. Modern Methods in Scientific Computing and Applications NATO Science Series II, Springer, Germany, 2002, 75: 255-281.

[13] Gao J W. An extended CEV model and the Legendre transform dual asymptotic solutions for annuity contracts. Insurance: Mathematics and Economics, 2010, 46(3): 511-530.

[14] Heston S L. A close-form solution for options with stochastic volatility with applications to bond and currency options. Review of Financial Studies, 1993, 6(2): 327-343.

[15] Li Z F, Zeng Y, Lai Y Z. Optimal time-consistent investment and reinsurance strategies for insurers under Heston's SV model. Insurance: Mathematics and Economics, 2012, 50(1): 191-203.

[16] Yi B, Li Z F, Viens F G, et al. Robust optimal control for an insurer with reinsurance and investment under Heston's stochastic volatility model. Insurance: Mathematics and Economics, 2013, 53(3): 601-614.

[17] Zhao H, Rong X M, Zhao Y G. Optimal excess-of-loss reinsurance and investment problem for an insurer with jump-diffusion risk process under the Heston model. Insurance: Mathematics and Economics, 2013, 53(3): 504-514.

[18] Li D P, Rong X M, Zhao H. Optimal reinsurance and investment problem for an insurer and a reinsurer with jump-diffusion risk process under the Heston model. Computational and Applied Mathematics, 2014, 53(3): 1-25.

[19] A C X, Li Z F. Optimal investment and excess-of-loss reinsurance problem with delay for an insurer under Heston's SV model. Insurance: Mathematics and Economics, 2015, 61(1): 181-196. 